\title{
Reemplazo valvular aórtico con prótesis biológica en pacientes mayores de 70 años con estenosis aórtica
}

\author{
RICARDO ZALAQUETT S., GABRIEL OLIVARES R., \\ RICARDO MEJÍA M. ${ }^{\mathrm{a}}$, FELIPE QUEZADA D. ${ }^{\mathrm{a}}$, OSLANDO PADILLA P. ${ }^{\mathrm{b}}$, \\ PEDRO BECKER R., MANUEL IRARRÁZAVAL LL., SERGIO MORÁN V., \\ CECILIA MUÑOZ C., RODRIGO GONZÁLEZ F.
}

División de Enfermedades

Cardiovasculares. Facultad de Medicina. Pontificia Universidad Católica de Chile. Santiago, Chile. anterno, Escuela de Medicina.

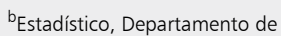
Salud Pública.

Recibido el 8 de junio de 2010, aceptado el 10 de diciembre de 2010.

Correspondencia a:

Dr. Ricardo Zalaquett S. Departamento de Enfermedades Cardiovasculares Pontificia Universidad Católica de Chile Marcoleta $367,8^{\circ}$ piso. Teléfono: 3543231 Fax: 6390108 Email: rzalaque@med.puc.cl

\section{Aortic valve replacement with bioprosthesis in patients 70 years old or older with aortic stenosis}

Background: Aortic valve stenosis is the most prevalent valvulopathy in the elderly, but it is frequently undertreated. Aim: To assess early and long term results of valve replacement with bioprostheses in patients 70 years old or older with aortic stenosis. Patients and Methods: A review of the database of the Cardiovascular Surgery Service of our hospital, for the period 1990-2007, identified 156 patients with aortic valve stenosis, aged $76.7 \pm 4.5$ years ( $67 \%$ males), that were subjected to an aortic valve replacement with bioprosthesis. Seventy eight (50\%) were in functional class III-IV. Six patients had emergency surgery. In 67 patients (43\%) coronary artery bypass graft were performed. Long term mortality was ascertained obtaining death records from Chilean death registry. Results: Operative mortality was 3.2\% (5 patients). Four patients $(2.6 \%)$ required a reoperation due to bleeding. The mean follow up was $66 \pm 41$ months. The survival rate at 1, 3 and 5 years was $94 \%, 88 \%$ and $80 \%$, respectively. Forty two patients (27\%) were 80 years old or older. In this sub-group, 1 patient (2.3\%) died during the peri operative period and the estimated survival rate at 1, 3 and 5 years was 97\%, 92\%, and 80\%, respectively. Multivariate risk analysis, for the whole group, identified low ejection fraction and concomitant coronary artery bypass graft surgery as predictors of late mortality. Conclusions: Aortic valve replacement for aortic stenosis with bioprostheses in patients 70 years old or older had low perioperative mortality and morbidity and a good long term survival.

(Rev Med Chile 2011; 139: 150-157).

Key words: Aged; Aortic valve stenosis; Bioprosthesis.
E l aumento de la expectativa de vida ha determinado un incremento de la población geriátrica ${ }^{1}$. En Chile, actualmente la esperanza de vida al nacer es de 77 años. Por otra parte, en 2005 los mayores de 65 años representaron 8\% y hacia 2025 representarán 14\% de la población general $^{2}$.

La prevalencia de estenosis aórtica en pacientes de edad avanzada es de $20 \%$, constituyendo la alteración estructural cardiaca más frecuente ${ }^{3}$.
Las estrategias terapéuticas conservadoras en este grupo de pacientes, como el manejo médico o la balonplastía, han demostrado mínimo alivio sintomático y malos resultados alejados ${ }^{4}$. Por el contrario, la evidencia actual demuestra resultados quirúrgicos, a corto y a largo plazo, muy satisfactorios para el reemplazo valvular aórtico con prótesis biológica, asociado a una buena calidad de vida $^{5-7}$. Sin embargo, se estima que un número importante de pacientes mayores con estenosis 
aórtica significativa no son derivados a cirugía para un reemplazo valvular aórtico, entre otras razones, probablemente, por desconocimiento de estos resultados ${ }^{8}$.

Recientemente, nuestro grupo reportó los resultados del reemplazo valvular aórtico aislado, ya sea por estenosis y/o insuficiencia aórtica, tanto con prótesis mecánica como biológica, demostrando una baja morbimortalidad operatoria y buenos resultados alejados, comparables a otras series".

En esta oportunidad, el objetivo fue evaluar específicamente los resultados inmediatos y a largo plazo del reemplazo valvular aórtico con prótesis biológica por estenosis aórtica, con o sin cirugía coronaria asociada, en pacientes de 70 años o más, en los que este tipo de prótesis tiene su indicación más precisa, ya que hay una menor incidencia de deterioro estructural protésico y el tratamiento anticoagulante es más engorroso y con mayor morbilidad secundaria.

\section{Pacientes y Método}

\section{Método}

Se revisó la base de datos del Servicio de Cirugía Cardiovascular del Hospital Clínico de la Pontificia Universidad Católica de Chile, para el período comprendido entre diciembre de 1990 y agosto de 2007. Se analizaron las características clínicas pre-operatorias y quirúrgicas, las complicaciones post-operatorias y la mortalidad operatoria (intrahospitalaria o hasta 30 días de la cirugía). La mortalidad alejada se obtuvo del Registro Civil e Identificación de Chile.

\section{Pacientes}

Entre diciembre 1990 y agosto 2007, 156 pacientes consecutivos de 70 años o más fueron sometidos a reemplazo valvular aórtico con prótesis biológica por estenosis aórtica, con o sin cirugía de revascularización miocárdica concomitante. Estos corresponden al $74 \%$ de los pacientes mayores de 70 años operados por estenosis aórtica en el período en estudio. Se excluyeron del análisis aquellos pacientes con insuficiencia aórtica predominante, endocarditis infecciosa, cirugía concomitante de otras válvulas o cirugía de la aorta torácica.

La Tabla 1 resume las características clínicas y quirúrgicas de los pacientes.

La edad promedio fue $76,7 \pm 4,5$ años. Ciento- cuatro pacientes $(66,6 \%)$ eran hombres. Setenta $\mathrm{y}$ ocho pacientes $(50 \%)$ estaban en capacidad funcional III-IV. Cincuenta y nueve pacientes (37\%) presentaron angina y 11 (7\%) síncope. Seis pacientes $(3,8 \%)$ fueron operados de urgencia. El EuroSCORE ${ }^{10}$ logístico promedio fue de 8,1 $\pm 5,2$ [El EuroSCORE logístico es usado para la estratificación de riesgo de los pacientes y para la estimación pre-operatoria de la mortalidad. Un EuroSCORE logístico < de 10\% es considerado de bajo riesgo; en cambio si es $>$ de $20 \%$ es considerado de alto riesgo ${ }^{11}$.

\section{Estadística}

Los resultados son expresados como promedio \pm desviación estándar. El análisis bivariado para las variables nominales fue mediante el test de Chi-cuadrado o en su lugar el test de probabilidad exacta de Fischer. Para variables continuas se utilizó una Prueba T o en su lugar la prueba no paramétrica de Mann-Whitney. En el análisis se incluyeron las siguientes variables: Características clínicas (Tabla 1) y quirúrgicas (Tabla 2), morbi-

Tabla 1. Características clínicas de 156 pacientes con reemplazo valvular aórtico con prótesis biológica por estenosis aórtica

\begin{tabular}{|c|c|c|}
\hline & $\mathbf{n}$ & $\%$ \\
\hline Pacientes & 156 & \\
\hline Edad (promedio \pm DS) & $76 \pm 4,5$ & \\
\hline Hombres & 104 & 66,6 \\
\hline CF III * & 43 & 27 \\
\hline CF IV & 35 & 22 \\
\hline $\mathrm{FE}<50 \% * *$ & 47 & 30 \\
\hline Hipertensión arterial & 81 & 51,9 \\
\hline Dislipidemia & 59 & 37,8 \\
\hline Tabaquismo & 33 & 21 \\
\hline Diabetes mellitus & 26 & 16,6 \\
\hline Infarto miocardio antiguo & 12 & 7,7 \\
\hline Fibrilación auricular & 8 & 5,1 \\
\hline Insuficiencia renal crónica & 8 & 5,1 \\
\hline Reoperación (esternotomía previa) & 7 & 4,5 \\
\hline Superficie Corporal (promedio \pm DS) & $1,3 \pm 0,8$ & \\
\hline EuroSCORE logístico (promedio \pm DS) & $8,1 \pm 5,2$ & \\
\hline
\end{tabular}

*CF Capacidad funcional / **FE Fracción de eyección 
Tabla 2. Características quirúrgicas de 156 pacientes con reemplazo valvular aórtico con prótesis biológica por estenosis aórtica

\begin{tabular}{|c|c|c|}
\hline & $\mathbf{n}$ & $\%$ \\
\hline \multicolumn{3}{|l|}{ Morfología válvula } \\
\hline Calcificación senil & 108 & 69 \\
\hline Válvula bicúspide & 48 & 31 \\
\hline \multicolumn{3}{|l|}{ Tipo prótesis } \\
\hline Carpentier-Edwards Pericardio & 106 & 68 \\
\hline Carpentier-Edwards Porcina & 42 & 27 \\
\hline Mitroflow & 3 & 1,9 \\
\hline Toronto SPV & 3 & 1,9 \\
\hline St. Jude BioCor & 2 & 1,2 \\
\hline Mediana tamaño valvular protésico & $23(19-27)$ & \\
\hline Cirugía revascularización miocárdica asociada & 67 & 43 \\
\hline Número puentes por paciente (promedio \pm DS) & $2,2 \pm 0,9$ & \\
\hline Número de injerto con arteria mamaria izquierda & 19 & 28 \\
\hline Tiempo circulación extracorpórea (min) (promedio \pm DS) & $133 \pm 39$ & \\
\hline Tiempo pinzamiento aórtico (min) (promedio \pm DS) & $99 \pm 30$ & \\
\hline
\end{tabular}

Tabla 3. Mortalidad operatoria y alejada de 156 pacientes con reemplazo valvular aórtico con prótesis biológica por estenosis aórtica

\begin{tabular}{|lrc|}
\hline & n & \% \\
Mortalidad operatoria & 5 & 3,2 \\
Insuficiencia cardiaca & 3 & \\
Mediastinitis & 1 & \\
Neumonía & 1 & \\
Mortalidad alejada & 44 & 28 \\
Causa no cardiaca & 29 & 66 \\
Neoplasias & 9 & \\
Neumonía & 9 & \\
Accidente vascular encefálico & 5 & \\
Insuficiencia renal & 3 & \\
Otras & 3 & \\
Causa cardiaca & 15 & 34 \\
Endocarditis & 6 & \\
Infarto miocardio & 5 & \\
Insuficiencia cardiaca & 4 & \\
\hline
\end{tabular}

lidad postoperatoria (Tabla 3) y las variables del EuroSCORE ${ }^{10,11}$.

Para el análisis multivariado se empleó el modelo de regresión logística para identificar los predictores de mortalidad y método de Kaplan-Meier y regresión de Cox para modelar la supervivencia. Sólo las variables que fueron significativas están en los resultados. Un valor $p$ menor de 0,05 se consideró significativo en todos los casos.

\section{Resultados}

\section{Características clínicas y quirúrgicas}

Se resumen en las Tablas 1 y 2 . Doce pacientes $(7,7 \%)$ tenían antecedentes de un infarto antiguo y 8 pacientes $(5,1 \%)$ estaban en fibrilación auricular persistente. En la mayoría de los casos (69\%) la válvula aórtica era de 3 velos con calcificación de tipo senil. En 106 pacientes (68\%) se utilizó una prótesis valvular de pericardio bovino CarpentierEdwards ${ }^{\circledR}$ Perimount y en 42 (27\%) una prótesis aórtica porcina, también Capentier Edwards ${ }^{\circledR}$. La mediana de tamaño valvular protésico fue $23 \mathrm{~mm}$. En 67 pacientes (43\%) se efectuó una revasculari- 
zación miocárdica asociada. El promedio de días de hospitalización fue $13 \pm 9$ días y la mediana fue 10 días (6-54).

\section{Mortalidad operatoria}

La mortalidad operatoria fue $3,2 \%$ (5 pacientes). Para el subgrupo con revascularización miocárdica asociada fue $4,4 \%$ ( 3 pacientes) y para los sin cirugía coronaria fue $2,2 \%$. Las causas de mortalidad se detallan en la Tabla 3.

\section{Morbilidad perioperatoria}

La Tabla 4 resume la morbilidad. La complicación más frecuente fue fibrilación auricular en 69 pacientes $(39,7 \%)$. Nueve pacientes $(5,7 \%)$ fueron reoperados en el período postoperatorio ( 5 por mediastinitis y 4 por hemorragia). Tres pacientes $(1,9 \%)$ presentaron un accidente cerebrovascular.

\section{Mortalidad alejada}

El promedio de seguimiento para supervivencia alejada fue de $66 \pm 41$ meses y la mediana 65 meses (1-159). Este se completó en el 98\% de los casos. En total, 44 pacientes fallecieron durante el seguimiento. De éstos, veintinueve $(66 \%)$ fallecieron de causa no cardiaca y $15(34 \%)$ de causa cardiaca (Tabla 3). La probabilidad actuarial de estar vivo a 1,3 y 5 años fue de $94 \%, 88 \%$ y $80 \%$, respectivamente (Figura 1). La probabilidad de estar libre de muerte cardiaca a 1, 3 y 5 años fue de $96 \%, 93 \%$ y $90 \%$, respectivamente (Figura 2).

\section{Reoperación valvular}

Sólo una paciente fue reoperada a los 53 días de su operación por una endocarditis protésica asociada a un pseudoaneurisma de aorta ascendente, efectuándose un reemplazo de ésta y de la prótesis biológica, con un homoinjerto criopreservado.

Tabla 4. Morbilidad post-operatoria de 156 pacientes con reemplazo valvular aórtico con prótesis biológica por estenosis aórtica

\begin{tabular}{|lcc|}
\hline & n & \% \\
\hline Fibrilación auricular & 62 & 39,7 \\
Neumonía & 9 & 5,7 \\
Marcapasos por bloqueo AV* & 7 & 4,4 \\
Reoperación por mediastinitis & 5 & 3,2 \\
Reoperación por hemorragia & 4 & 2,5 \\
Accidente vascular encefálico & 3 & 1,9 \\
Convulsiones & 2 & 1,2 \\
Balón contrapulsación intra-aórtico & 2 & 1,2 \\
\hline
\end{tabular}

*AV auriculoventricular.

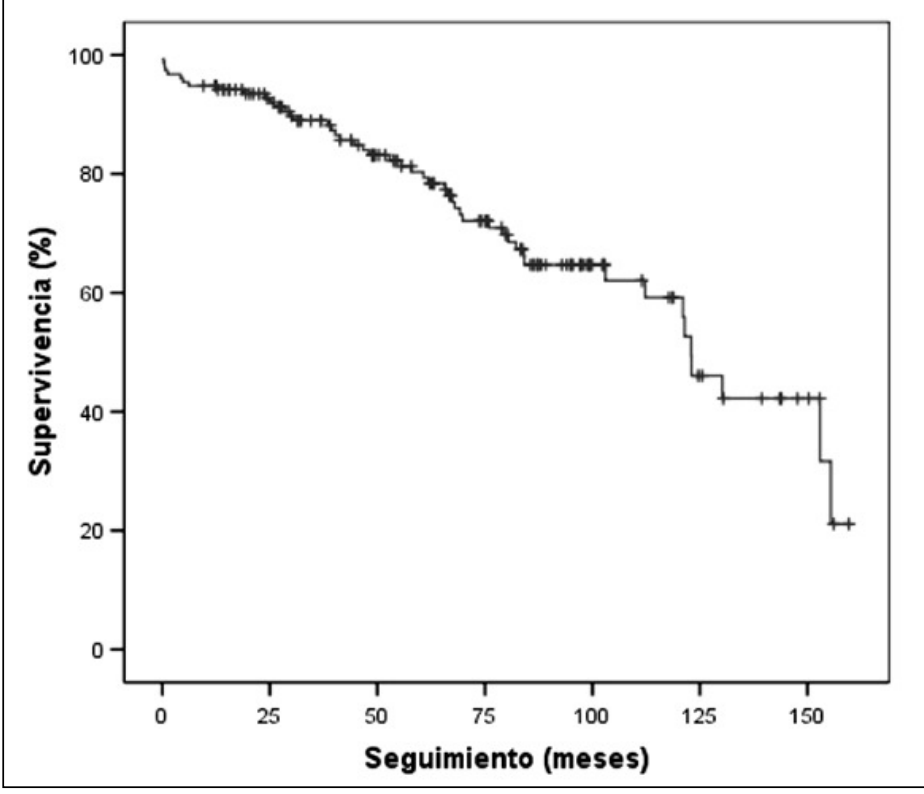

Figura 1. Curva de Kaplan-Meier de supervivencia global para pacientes mayores de 70 años con reemplazo valvular aórtico por estenosis aórtica con prótesis biológica. 


\section{Octogenarios}

Cuarenta y dos pacientes (27\%) tenían 80 años o más. En este subgrupo un paciente (2,3\%) falleció en el periodo perioperatorio. En total, 14 pacientes fallecieron durante el seguimiento. La probabilidad de estar vivo para éstos a 1, 3 y
5 años fue de 97\%, 92\% y $80 \%$, respectivamente (Figura 3).

\section{Análisis estadístico}

El análisis bivariado identificó sólo a la angina como predictor de mortalidad operatoria. Por
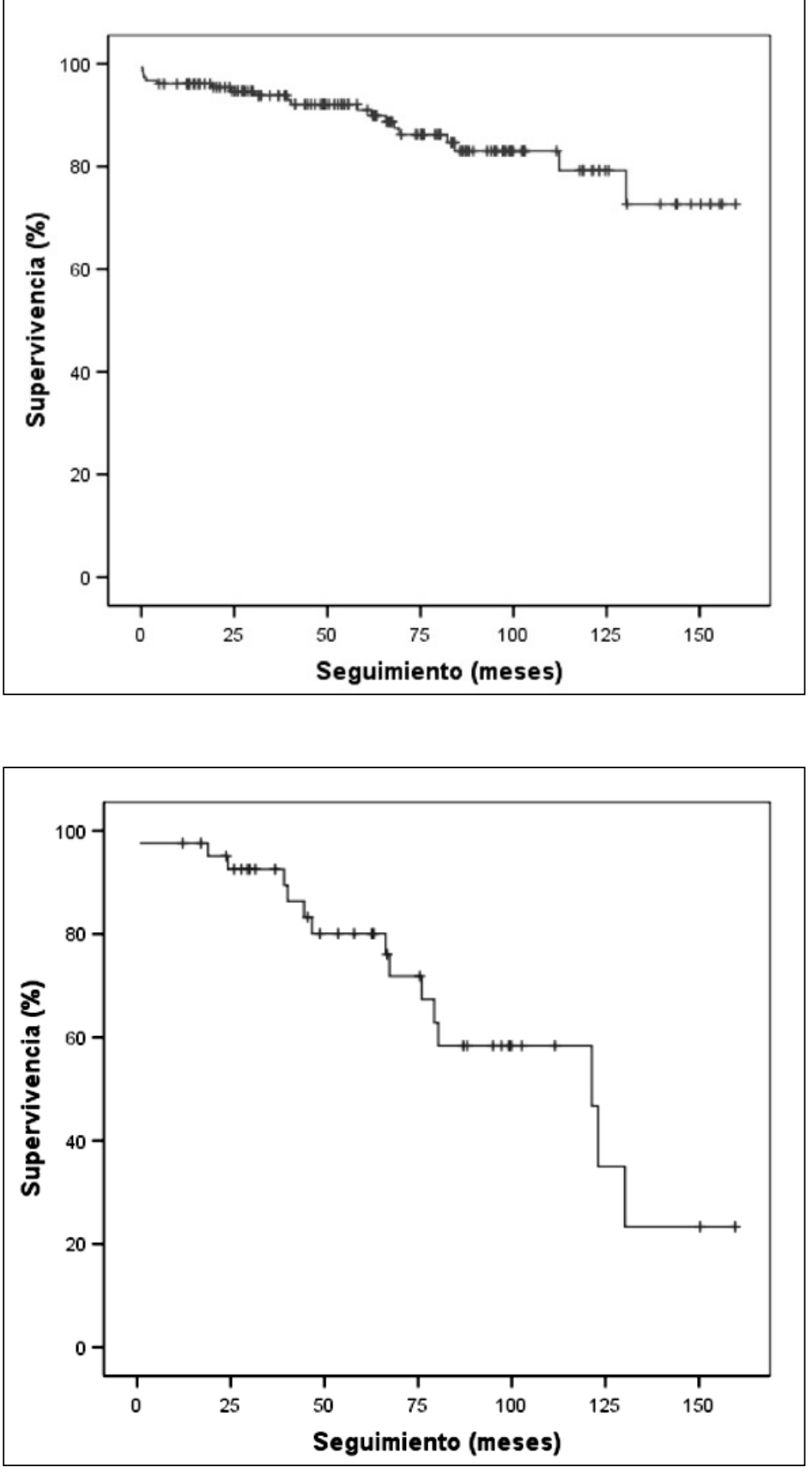

Figura 2. Curva de Kaplan-Meier de supervivencia alejada libre de muerte cardiaca para pacientes mayores de 70 años con reemplazo valvular aórtico por estenosis aórtica con prótesis biológica.

Figura 3. Curva de Kaplan-Meier de supervivencia alejada para pacientes mayores de 80 años con reemplazo valvular aórtico por estenosis aórtica con prótesis biológica. 
Tabla 5. Análisis bivariado para mortalidad operatoria y alejada de 156 pacientes con reemplazo valvular aórtico con prótesis biológica por estenosis aórtica

\begin{tabular}{|c|c|c|c|c|c|}
\hline & & \multicolumn{4}{|c|}{ Mortalidad operatoria } \\
\hline & & No & & Sí & Valor $\mathbf{p}$ \\
\hline \multirow[t]{4}{*}{ Angina } & No & 95 & 0 & $(0 \%)$ & 0,022 \\
\hline & Sí & 57 & 4 & $(6,6 \%)$ & \\
\hline & & \multicolumn{4}{|c|}{ Mortalidad alejada } \\
\hline & & No & & Sí & Valor $\mathbf{p}$ \\
\hline \multirow[t]{2}{*}{ Revascularización miocárdica asociada } & No & 70 & 20( & $(22,2 \%)$ & \multirow[t]{2}{*}{0,004} \\
\hline & Sí & 37 & 29( & $(43,9 \%)$ & \\
\hline Disfunción ventrículo izquierdo & No & 91 & 32( & $(26 \%)$ & 0,005 \\
\hline$(\mathrm{FE}<50 \%)^{*}$ & Sí & 16 & 17( & $(51,5 \%)$ & \\
\hline \multirow[t]{2}{*}{ Prótesis Carpentier-Edwards Porcina } & No & 86 & 23( & $(21,1 \%)$ & \multirow[t]{2}{*}{$<0,0001$} \\
\hline & Sí & 18 & 25( & $(58,1 \%)$ & \\
\hline
\end{tabular}

*FE Fracción de eyección.

Tabla 6. Análisis multivariado para mortalidad alejada de 156 pacientes con reemplazo valvular aórtico con prótesis biológica por estenosis aórtica

\begin{tabular}{|lccc|}
\hline & Odds ratio & $\mathbf{9 5 \%}$ IC** & Valor p \\
\hline Disfunción del Ventrículo Izquierdo $(\mathrm{FE}<50 \%)^{*}$ & 2,38 & $1,31-4,34$ & 0,004 \\
\hline Revascularización Miocárdica Asociada & 2,19 & $1,23-3,9$ & 0,008 \\
\hline
\end{tabular}

*FE Fracción de eyección / **IC Intervalo de confianza.

otra parte, durante el seguimiento este análisis identificó a la revascularización miocárdica asociada, la disfunción del ventrículo izquierdo y a la utilización de una prótesis valvular porcina como predictor de mortalidad alejada (Tabla 5). El análisis multivariado sólo identificó a la disfunción del ventrículo izquierdo y a la revascularización miocárdica asociada como predictor de mortalidad alejada (Tabla 6).

\section{Discusión}

La historia natural de la estenosis aórtica es mala; $90 \%$ de los pacientes con angina y síncope mueren a los 3 años del comienzo de los síntomas y si la falla cardiaca esta presente la muerte ocurre, en promedio, a los 2 años $^{12}$. El tratamiento médico esta igualmente asociado con malos resultados, con una supervivencia a 5 y 10 años de $32 \%$ y $18 \%$, respectivamente ${ }^{13}$. Finalmente, la balonplastía aórtica percutánea es de uso limitado en la actualidad, debido a los resultados hemodinámicos discretos y a la alta incidencia de re-estenosis, reservándose su indicación como puente a la cirugía o de forma paliativa en pacientes con muy elevado riesgo quirúrgico o portadores de una enfermedad terminal ${ }^{4}$. Por otro lado, la estenosis aórtica es una patología frecuente del adulto mayor, para los cuales el reemplazo valvular aórtico con prótesis biológica es el tratamiento más efectivo en la actualidad, pero un número significativo de estos no son, en la práctica, derivados a cirugía, lo que en parte podría deberse a falta de información adecuada de los resultados quirúrgicos inmediatos y alejados de esta ${ }^{3,8}$. 


\section{Mortalidad operatoria}

En nuestro estudio, la mortalidad operatoria fue de $3,2 \%$ y para los pacientes mayores de 80 años de 2,3\%; en los pacientes con revascularización miocárdica asociada fue de 4,4\% y en los sin cirugía coronaria fue de 2,2\%. Estas cifras son comparables con las reportadas recientemente. Así, trabajos actuales comunican una mortalidad operatoria en pacientes mayores sometidos a reemplazo valvular aórtico aislado entre 3,9\% y $4,1 \%{ }^{14,15}$ y para la cirugía combinada de reemplazo valvular aórtico y revascularización miocárdica entre $3 \%$ y $5,6 \%{ }^{15,16}$.

\section{Morbilidad perioperatoria}

Las complicaciones post-operatorias en nuestra serie (Tabla 4) en general son similares a otras publicaciones. Específicamente, en publicaciones recientes, la incidencia de accidente vascular encefálico varía entre $2 \%$ a $5,4 \% 5,6,14$, reoperación por hemorragia entre $4 \%$ a $10 \%{ }^{5,6,14}$, mediastinitis entre $1 \%$ a $2,3 \%{ }^{5,6,14}$, implante de marcapasos entre $4,1 \%$ a $5 \%{ }^{6,14}$ y fibrilación auricular entre $37 \%$ a $39,8 \%{ }^{5,14}$.

\section{Supervivencia alejada}

En nuestra serie la supervivencia a 1, 3 y 5 años fue $94 \%, 88 \%$ y $80 \%$, respectivamente. Esta supervivencia fue similar a otras series publicadas, como la de Mihaljevic et al, de la Cleveland Clinic $^{16}$, quienes comunicaron 3.049 pacientes con estenosis aórtica sometidos a reemplazo valvular con prótesis biológica, con un promedio de edad de 73 años y $67 \%$ de revascularización miocárdica asociada. En ésta, la supervivencia a 1 y 5 años fue de $91 \%$ y $75 \%$, respectivamente. Las principales causas de muerte fueron no cardiacas (66\% de los casos), siendo las más frecuentes las neoplasias y las neumonías, lo que en parte se explica por la edad avanzada de los pacientes.

Igualmente, la supervivencia en pacientes octogenarios, en nuestra experiencia, fue comparable a otras series publicadas, las que reportan cifras de alrededor de $90 \%$ al año y de $80 \%$ a 5 años ${ }^{6,7}$.

En nuestro trabajo, las variables asociadas a mayor mortalidad alejada en el análisis bivariado fueron la disfunción del ventrículo izquierdo, la revascularización miocárdica asociada y la utilización de una válvula protésica porcina. En el análisis multivariado sólo resultaron asociados la disfunción del ventrículo izquierdo y la revascularización miocárdica.

Hannan et $\mathrm{al}^{15}$ publicaron un estudio que incluyó 6.369 pacientes con estenosis aórtica, con o sin revascularización miocárdica, determinando, igualmente, que los factores de riesgo de mortalidad a 30 meses fueron la revascularización miocárdica asociada y una baja fracción de eyección, lo que es también concordante con la experiencia de Mihaljevic et al $^{16}$.

Respecto al tipo de prótesis biológica, Jamieson et $\mathrm{al}^{17}$ compararon los resultados clínicos entre la prótesis de porcino y la de pericardio bovino. La mortalidad alejada fue $51 \%$ para la primera versus $25 \%$ para la segunda, lo que es también concordante con nuestro estudio, si bien los pacientes con prótesis porcina se concentran al inicio de nuestra experiencia y en la serie de Jamieson éstos tenían una mayor incidencia de cirugía coronaria asociada.

\section{Limitaciones del estudio}

El trabajo tiene las limitaciones propias de todo estudio observacional y descriptivo, por lo que no permite comparaciones absolutamente válidas con otras alternativas de tratamiento. Otra limitante es que el seguimiento sólo consideró la supervivencia alejada de los pacientes. Específicamente, no se pudo obtener información respecto a deterioro estructural de las prótesis, lo que hubiera requerido de un ecocardiograma al cierre del seguimiento de los sobrevivientes, sin poder precisar las condiciones de las prótesis en los fallecidos durante éste.

\section{Conclusión}

La cirugía de reemplazo valvular con prótesis biológica por estenosis aórtica en pacientes de 70 años o más tiene baja mortalidad y morbilidad perioperatoria y buenos resultados alejados.

\section{Implicancias del estudio}

Estos buenos resultados, inmediatos y alejados, del reemplazo valvular aórtico con prótesis biológica en pacientes mayores portadores de estenosis aórtica debieran llevar a no considerar la edad por sí misma como factor de riesgo y hacer que un número cada vez mayor de pacientes se beneficien de la cirugía. Igualmente, estos resultados debieran considerarse como un referente cuando 
Reemplazo valvular aórtico en mayores de 70 años - R. Zalaquett et al

los procedimientos percutáneos de reemplazo valvular aórtico de reciente introducción, hasta ahora reservados para pacientes considerados como contraindicados para la cirugía, se comiencen a aplicar en pacientes de menor riesgo quirúrgico, que, como señala un reciente y autorizada editorial, es esperable que pronto suceda ${ }^{18}$.

\section{Referencias}

1. Compendio de Estadísticas Vitales, Instituto Nacional de Estadísticas de Chile (INE) 2006: 4-5.

2. Enfoque Estadístico, INE de Chile, junio 2004. Disponible en: http://www.ine.cl/canales/sala_prensa/ noticias/2004/jun/not250604.php. Consultado el 17 de enero de 2009.

3. Aronow WS, Kronzon I. Prevalence and severity of valvular aortic stenosis determined by Doppler echocardiography and its association with echocardiographic and electrocardiographic left ventricular hypertrophy and physical signs of aortic stenosis in elderly patients. Am J Cardiol 1991; 67: 776-7.

4. Bonow RO, Carabello BA, Chatterjee K, de Leon AC Jr, Faxon DP, Freed MD, et al. ACC/AHA 2006 guidelines for the management of patients with valvular heart disease: a report of the American College of Cardiology/ American Heart Association Task Force on Practice Guidelines (writing Committee to Revise the 1998 guidelines for the management of patients with valvular heart disease) developed in collaboration with the Society of Cardiovascular Anesthesiologists endorsed by the Society for Cardiovascular Angiography and Interventions and the Society of Thoracic Surgeons. Circulation 2006; 114: e84-231.

5. Sundt TM, Bailey MS, Moon MR, Mendeloff EN, Huddleston CB, Pasque MK, et al. Quality of life after aortic valve replacement at the age of 80 years. Circulation 2000; 102: 70-4.

6. Kolh P, Kerzmann A, Honore C, Comte L, Limet R. Aortic valve surgery in octogenarians: predictive factors for operative and long-term results. Eur J Cardiothorac Surg 2007; 31: 600-6.

7. Chiappini B, Camurri N, Loforte A, Di Marco L, Di Bartolomeo R, Marinelli G. Outcome after aortic valve replacement in octogenarians. Ann Thorac Surg 2004;
78: 85-9.

8. Van Geldorp MW, van Gamerena M, Kappeteina AP, Arabkhania B, de Groot-de Laatb LE, Takkenberga J, et al. Therapeutic decisions for patients with symptomatic severe aortic stenosis: room for improvement? Eur J Cardiothorac Surg 2009; 35: 953-7.

9. Becker P, Ramírez A, Cifuentes I, Rebolledo R, Zalaquett $\mathrm{R}$, Moran S, et al. [Early and late morbidity and mortality of aortic valve replacement with mechanical or biological prostheses during the last decade: The gold standard]. Rev Med Chile 2009; 137: 1153-62.

10. Roques F, Nashef SA, Michel P, Gauducheau E, de Vicentiis C, Baudet E, et al. Risk factors and outcome in European cardiac surgery: analysis of the EuroSCORE multinational database of 19030 patients. Eur J Cardiothorac Surg 1999; 15: 816-22.

11. Leontyev S, Walther T, Borger MA, Lehmann S, Funkat $\mathrm{AK}$, Rastan A, et al. Aortic valve replacement in octogenarians: utility of risk stratification with EuroSCORE. Ann Thorac Surg 2009; 87:1440-5.

12. Frank S, Johnson A, Ross J. Natural history of valvular aortic stenosis. Br Heart J 1973; 35: 41-6.

13. Varadarajan P, Kapoor N, Bansal RC, Pai RG. Clinical profile and natural history of 453 nonsurgically managed patients with severe aortic stenosis. Ann Thorac Surg 2006; 82: 2111-5.

14. Thourani VH, Myung R, Kilgo P, Thompson K, Puskas JD, Lattouf OM, et al. Long-term outcomes after isolated aortic valve replacement in octogenarians: a modern perspective. Ann Thorac Surg 2008; 86: 1458-65.

15. Hannan EL, Samadashvili Z, Lahey SJ, Smith CR, Culliford AT, Higgins R, et al. Aortic valve replacement for patients with severe aortic stenosis: risk factors and their impact on 30-month mortality. Ann Thorac Surg 2009; 87: 1741-50.

16. Mihaljevic T, Nowicki ER, Rajeswaran J, Blackstone EH, Lagazzi L, Thomas J, et al. Survival after valve replacement for aortic stenosis: implications for decision making. J Thorac Cardiovasc Surg 2008; 135: 1270-79.

17. Jamieson E, Germann E, Aupart M, Neville P, Marchand M, Fradet G. 15-year comparison of supra-annular porcine and perimount aortic bioprostheses. Asian Cardiovasc Thorac Ann 2006; 14: 200-5.

18. Webb J. Transcatheter Aortic Valve Implantation. Balancing Enthusiasm and Caution. Rev Esp Cardiol 2010; 63: 131-3. 